\title{
Eigenständige Regionalentwicklung als Strategie für periphere ländliche Räume? \\ Eine Untersuchung aus dem Schweizer Berggebiet
}

\author{
Sabine Mühlinghaus, Zürich
}

\section{Einleitung}

\subsection{Strukturwandel im Berggebiet}

Die Schweiz ist als kleine, offene Volkswirtschaft eng mit dem weltwirtschaftlichen Strukturwandel verflochten. Sie ist deshalb von den Veränderungen, welche die Globalisierung mit sich bringt, stark betroffen. In vielen Bergregionen dominieren strukturschwache Wirtschaftszweige wie die Landwirtschaft, binnenorientierte Industrie- und Gewerbebetriebe sowie das Gastgewerbe. Sie sind dem erhöhten Wettbewerbsdruck, welcher mit der Globalisierung einhergeht, kaum gewachsen. Eine besondere Herausforderung für das Schweizer Berggebiet stellt die Neuorientierung der Schweizer Agrarpolitik dar, aufgrund welcher mit einem Verlust von Arbeitsplätzen zu rechnen ist. Auch die Regionalpolitik ist neu vermehrt nach Effizienzzielen ausgerichtet, was für die strukturschwächsten Bergregionen nicht unproblematisch ist. Zusätzlich findet gegenwärtig in verschiedenen Bereichen der Grundversorgung ein Abbau staatlicher Regulierungen statt, welcher sowohl die Versorgung peripherer Regionen in Frage stellt, als auch zu einem Abbau von Arbeitsplätzen in Randregionen führt. Für Regionen, welche weder im Einzugsbereich grösserer Agglomerationen liegen, noch über eine konkurrenzfähige touristische Infrastruktur verfügen, stellen diese Veränderungen eine zunehmende Bedrohung dar. In diesem Artikel soll aufgezeigt werden, inwiefern eigenständige Regionalentwicklung solchen peripheren Regionen eine Chance bietet, um sich unter diesen veränderten Bedingungen zu behaupten.

\subsection{Globalisierung oder die Renaissance eigen- ständiger Regionalentwicklung}

Das Aufkommen des Konzeptes der eigenständigen Regionalentwicklung ist im Zusammenhang mit Strategiediskussionen in Entwicklungsländern zu sehen, wobei dort eher Begriffe wie Dritter Weg oder SelfReliance verwendet wurden (HeINTEL 1998: 40-41). Gegen Ende der 1970er und in den 80er begann man auch in Europa vermehrt über alternative Konzepte der Regionalentwicklung zu diskutieren. Im deutschsprachigen Raum nimmt dabei Österreich eine Vorreiterrolle ein (Glatz \& Scheer 1981, Sauberer et al. 1989, STöHr 1981). Das Konzept wurde aber auch in der Schweiz (Brugger 1984, 1986, Bassand 1986,
ElSASSER 1987) und in Deutschland (Hahne 1985, HARTKE 1985) bald aufgegriffen.

In der zweiten Hälfte der 90er Jahre lässt sich ein Wiederaufleben der Thematik beobachten. Dies kann als Reaktion auf die Globalisierung interpretiert werden. Die Aufwertung des Kleinräumigen wird zunehmend als Chance betrachtet (vgl. z.B. BRYDEN 1998, FürST 2000, WILSON 1995). Zudem hat die Idee der eigenständigen Regionalentwicklung seit den 90er Jahren in regionalpolitischen Konzepten vermehrt Berücksichtigung gefunden, was zu verschiedenen praxisorientierten Arbeiten und Evaluationen angeregt hat (z.B. Barke \& Newton 1994, Bruckmeier 2000, Farrell et al. 1999, Ray 1998, 2000, Shucksmith 2000).

Aufgrund des ausgeprägten Föderalismus und der direkten Demokratie herrschen in der Schweiz günstige Voraussetzungen für eine eigenständige Regionalentwicklung. Dennoch sind aus der Schweiz bisher kaum Arbeiten bekannt, welche sich umfassend mit den Umsetzungsmöglichkeiten und Chancen dieses Konzeptes befassen. In vorliegendem Artikel werden basierend auf zwei Fallstudien aus dem Schweizer Berggebiet und Expertengesprächen die Umsetzungsprozesse, Erfolgsaussichten und Fördermöglichkeiten eigenständiger Regionalentwicklung erläutert (vgl. MüHLINGHAUS 2002).

\subsection{Methodik}

Die beiden Fallstudien - Urnäsch und Schamserberg - unterscheiden sich sowohl in ihrer Grösse als auch in ihrer Wirtschaftsstruktur, Tradition und in ihren Entwicklungsmöglichkeiten deutlich (vgl. Abbildung 1). Durch den Vergleich solcher kontrastierender Beispiele lässt sich erkennen, welches fallspezifische Elemente sind und welche auch unter veränderten Voraussetzungen auftreten.

Der Ansatz der eigenständigen Regionalentwicklung basiert auf der Wahrnehmung der handelnden Personen und ihren Zukunftsperspektiven. Um diesen subjektiven Sichtweisen gerecht zu werden, wurde ein qualitatives Vorgehen gewählt (FLICK 1996, LAMNEK 1995a, 1995b). Die Datenerhebung erfolgte mittels offener Interviews mit der Bevölkerung, lokalen Behörden und Experten. Dem Grundsatz des theoretischen Sampling folgend wurden während des Analyseprozesses fortlaufend und zielgerichtet neue Fälle in die Untersu- 


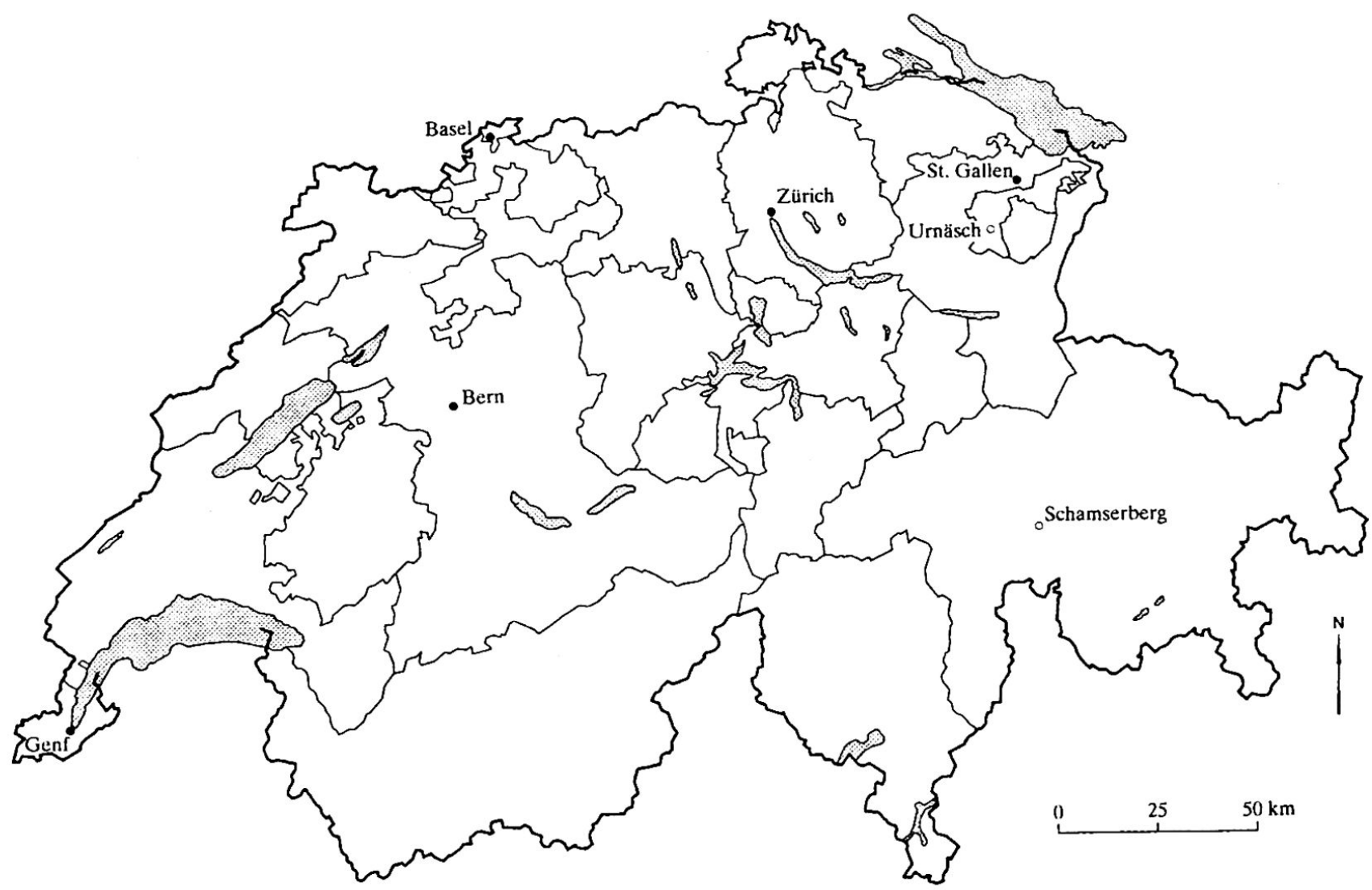

Abb. 1: Urnäsch und Schamserberg - Fallstudien aus dem Schweizer Berggebiet

Urnäsch and Schamserberg - Case studies from Swiss mountain areas

Urnäsch et Schamserberg - Etudes de cas dans les régions de montagne suisses

Quelle: MüHLINGHAus 2002: 38; Kartographie: V. SCHEURING

chung einbezogen, welche es erlaubten die theoretischen Konzepte zu vertiefen und zu differenzieren (Glaser \& Strauss 1998: 53-83). Die Auswertung der Interviews erfolgte in einer computerunterstützten qualitativen Inhaltsanalyse (MAYRING 2000), welche eine detaillierte Auswertung der Daten ermöglicht hat.

\section{Das Konzept der eigenständigen Regional- entwicklung}

Eigenständige Regionalentwicklung stellt kein in sich geschlossenes Konzept mit klaren theoretischen Wurzeln dar (Dujmovits 1996: 72, Mose 1989: 160). Vielmehr handelt es sich um eine Bündelung von Leitideen, welche einen idealisierten, deskriptiven Kontrast zu häufig beobachteten Entwicklungsmustern darstellen und durch Wertvorstellungen geleitet werden (SLEE 1994: 184/191). Nachfolgend werden die wichtigsten Ideen zusammengefasst, welche von verschiedenen Autoren aufgegriffen werden (vgl. z.B. Dusmovits
1996, Fürst 2000, Hahne 1985, 1987, HeinTel 1998, Hoffmann \& Nuissl 1998, Pongratz \& Kreil 1991).

Eigenständige Regionalentwicklung strebt eine selbstbestimmte, ganzheitliche Entwicklung an, welche auf der Aktivierung endogener Potentiale basiert (vgl. Abbildung 2). Darunter werden natürliche Ressourcen verstanden, genauso wie menschliche Fähigkeiten, soziokulturelle und wirtschaftliche Ressourcen. In politischer Hinsicht strebt eigenständige Regionalentwicklung eine Dezentralisierung von Macht und die Partizipation der Bevölkerung an politischen Prozessen an. Durch den Aufbau regionaler Wirtschaftskreisläufe und den gezielten Export hochwertiger Produkte soll die regionale Wirtschaft gestärkt werden. In soziokultureller Hinsicht geht es um die Förderung regionaler Identität und Kultur. Schliesslich sollen die Handlungsansätze vermehrt an die natürlichen Standortbedingungen angepasst werden und wo möglich und sinnvoll, auf regionale natürliche Ressourcen zurückgreifen. Zentrales Instrument der Umsetzung eigenständiger Regionalentwicklung sind lokale Initiativen. Darunter sind kooperative 


\begin{tabular}{|c|c|c|c|}
\hline & $\begin{array}{r}\text { Erhöhung der } \\
\text { Verminderung e } \\
\text { nachhaltiq }\end{array}$ & $\begin{array}{l}\text { ZIELE } \\
\text { er Selbstbestimmung } \\
\text { externer Abhängigkeit } \\
\text { ige Entwicklung }\end{array}$ & \\
\hline $\begin{array}{l}\text { Politik: } \\
\text {-Dezentralisierung } \\
\text { politischer Macht } \\
\text {-Partizipation der } \\
\text { lokalen Bevölke- } \\
\text { rung }\end{array}$ & $\begin{array}{l}\text { STR } \\
\text { Nutzung end } \\
\text { Wirtschaft: } \\
\text {-Aufbau regionaler } \\
\text { Wirtschaftskreisläufe } \\
\text {-Export hochwertiger } \\
\text { Produkte }\end{array}$ & $\begin{array}{l}\text { ATEGIEN } \\
\text { dogener Potentiale } \\
\text { Soziokultur: } \\
\text { I-Stärkung regionaler } \\
\text { Identität } \\
\text {-Förderung der } \\
\text { regionalen Kultur } \\
\end{array}$ & $\begin{array}{l}\text { Umwelt: } \\
\text {-Anpassung an na- } \\
\text { türliche Standort- } \\
\text { i voraussetzungen } \\
\text {-Nutzung regionaler } \\
\text { natürlicher Ressourcen }\end{array}$ \\
\hline \multicolumn{4}{|c|}{$\begin{array}{l}\text { UMSETZUNG } \\
\text { Lokale Initiativen }\end{array}$} \\
\hline
\end{tabular}

Abb. 2: Ziele und Strategien eigenständiger Regionalentwicklung

Aims and strategies of endogenous development

Buts et stratégies du développement endogène

Quelle: MühuINGhaus 2002: 25

und innovative Aktivitäten der lokalen Bevölkerung zu verstehen, welche die lokale Lebens-, Arbeits- und Umweltsituation verbessern (BIRNER et al. 1997: 31, STÖHR 1992: 32).

\section{Die Fallstudien}

Im Folgenden werden zwei Fallstudien vorgestellt, anhand derer untersucht wurde, wie eigenständige Regionalentwicklung die Situation strukturschwacher Gebiete, die einseitig auf die Landwirtschaft und Textilindustrie ausgerichtet waren, verbessern kann.

\subsection{Urnäsch - eine Textilgemeinde im Aufbruch}

Urnäsch ist eine voralpine Gemeinde mit 2370 Einwohner (1998). Trotz der Krise in der Textilindustrie arbeiteten 1995 noch immer $14 \%$ aller Beschäftigten in diesem Sektor (Schweiz 1\%). Ebenfalls deutlich übervertreten ist die Landwirtschaft mit einem Beschäftigtenanteil von $15 \%$ (Schweiz 3\%). Beide Sektoren haben in den letzten Jahren markante Einbussen erlebt, so dass die Gesamtzahl der Arbeitsplätze in Urnäsch zwischen 1985 und 1995 um 9\% gesunken ist (BUNDESAMT FüR StATistik 1998a, 1998b).

Lokale Initiativen haben in Urnäsch Tradition, weil die Gemeinde aufgrund ihrer angespannten finanziellen Lage schon seit langem eng begrenzte Möglichkeiten hatte. So gibt es seit 1967 eine Immobilien AG, welche sich um die Erschliessung und den Verkauf von Bau- land kümmert oder das Museum für Appenzeller Brauchtum, welches seit 1976 von einem Verein geführt wird. Für neue Dynamik hat das Projekt «Urnäsch - mitenand vorwärts» gesorgt. Dieses wurde 1996 mit einer Intensivwoche gestartet, während der Familien, Schulklassen und Interessengruppen von einem externen Team über ihre Ideen für die Zukunft der Gemeinde befragt wurden. Heute arbeiten Freiwillige in verschiedenen Arbeitsgruppen an der Umsetzung dieser Ideen. Auf diese Weise ist unter anderem ein Kulturverein entstanden, ein Dorfcafé eröffnet und eine Wirtschaftsförderungsgruppe gegründet worden.

Insgesamt konnten lokale Initiativen in Urnäsch einen wichtigen Beitrag zur Verbesserung der Lebensqualität und des sozialen Zusammenhalts leisten. Allerdings hat es sich als schwierig erwiesen, mit solchen lokalen Handlungsansätzen gegen die problematische wirtschaftliche Situation anzukommen.

\subsection{Schamserberg - Überleben mit der Berglandwirtschaft}

Im Schamserberg leben 390 Personen (1998) in fünf Gemeinden. Fast $60 \%$ der Bevölkerung sind in der Landwirtschaft tätig, weshalb die Gegend vom laufenden landwirtschaftlichen Strukturwandel stark betroffen ist (Bundesamt Für Statistik 1998a, 1998b). Einige lokale Initiativen streben Verbesserungen für die Landwirte an. So existiert eine Biobauerngenossenschaft, eine Vereinigung von Bauersfrauen, welche Geschenkpakete mit hausgemachten Produkten verkauft und 
eine Genossenschaft, welche den Bau eines regionalen Schlachthauses plant.

Die Einwohner des Schamserberges sind sich einig, dass man als Ergänzung zur Landwirtschaft den Tourismus fördern sollte. Hier hat die öffentliche Hand verschiedentlich Initiative ergriffen. Die Gemeinde Wergenstein hat das von der Schliessung bedrohte Hotel Piz Vizan gekauft und verpachtet, die Gemeinde Lohn hat den Bau eines Restaurants finanziert. Seit längerem besteht ein Projekt zur Erschliessung eines Wintersportgebietes. Dieses wird jedoch sehr kontrovers diskutiert und hat zu einem heftigen Konflikt zwischen Befürwortern und Gegnern geführt. Dieser hat die Kommunikation und Zusammenarbeit im Schamserberg erheblich erschwert. Aufgrund des Konflikts fehlt es an gemeinsamen Zielvorstellungen und somit auch an gemeinsamen Anstrengungen. Zusätzlich besteht infolge der einseitigen Wirtschaftsstruktur eine grosse Abhängigkeit von Subventionen und Spendegeldern, was den lokalen Handlungsspielraum reduziert und auf lokale Akteure oft entmutigend wirkt.

\section{Resultate}

\subsection{Einflüsse und Ressourcen in der Umsetzung eigenständiger Regionalentwicklung}

Aus der Analyse der Fallstudien geht hervor, dass nicht einzelne Faktoren, sondern das vielfältige Zusammen- spiel mehrerer Faktoren über den Erfolg lokaler Initiativen entscheidet (vgl. Abbildung 3).

Treibende Kraft eigenständiger Regionalentwicklung sind die lokalen Akteure; mit ihrer Handlungsbereitschaft und ihren Fähigkeiten steht und fällt die Umsetzung. Idealerweise bringen die Akteure neben innovativen Ideen Erfahrung und Know-how mit. Wichtig ist weiter, wie die Zusammenarbeit zwischen lokalen Akteuren und Behörden funktioniert und wie Akteure und Projekte von der Bevölkerung akzeptiert werden.

Obwohl eigenständige Regionalentwicklung primär ein regionaler Prozess ist, sind externe Ressourcen in Form von Finanzierungen und Beratungen unabdingbar. Diese externen Ressourcen können als Bestandteil des endogenen Prozesses betrachtet werden, weil sie von den lokalen Akteuren aktiv gesucht werden müssen.

Damit die internen und externen Ressourcen zum Tragen kommen, braucht es Interaktionen und Beziehungsverflechtungen sowohl innerhalb der Region als auch gegen aussen. Allen voran sind eine funktionierende Kommunikation und ein reger Informationsaustausch in der Bevölkerung sowie zwischen Bevölkerung und Behörden Voraussetzung dafür, dass ein gemeinsames Problembewusstsein und gemeinsame Handlungsstrategien entwickelt werden können. Sie fördern

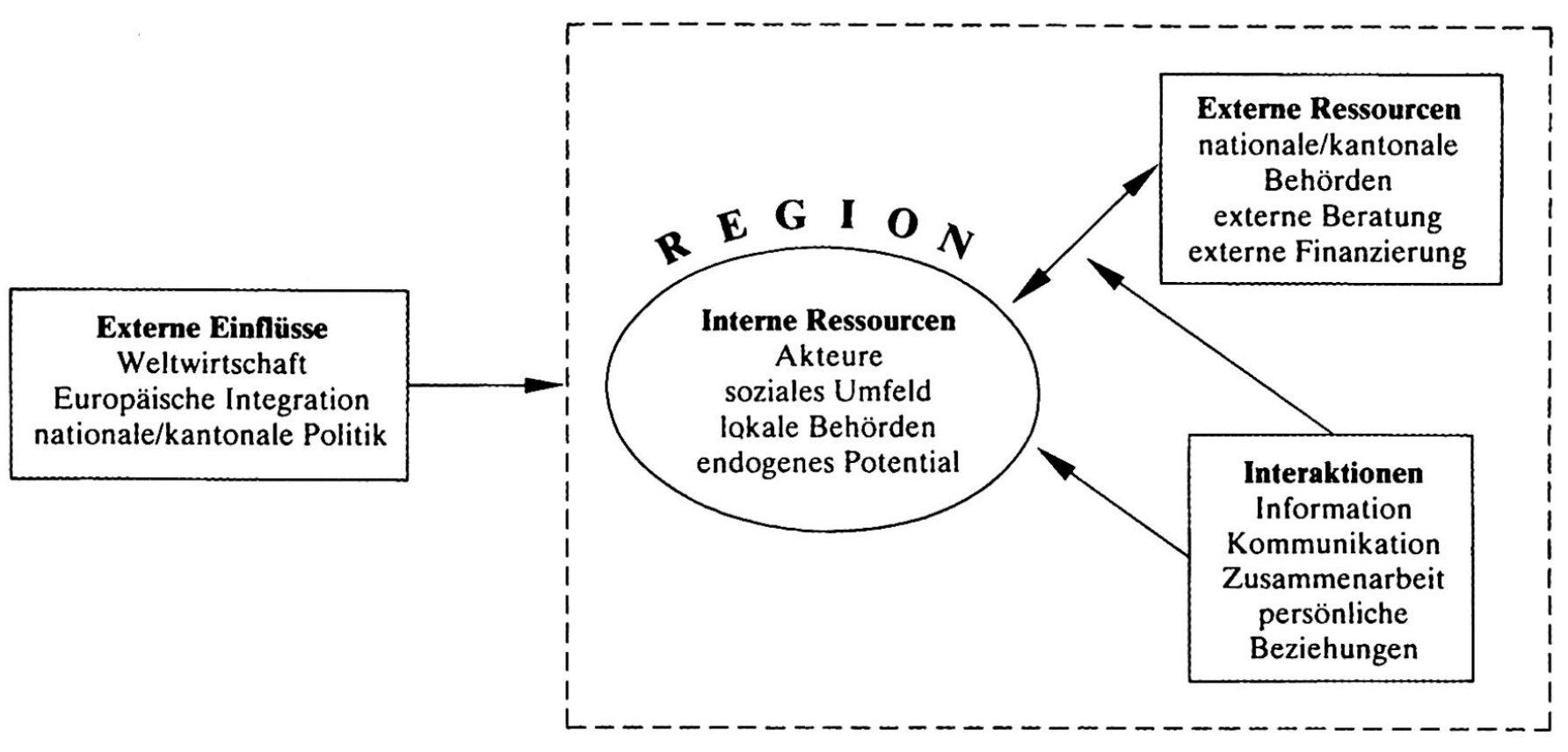

Abb. 3: Einflüsse und Ressourcen in der Umsetzung eigenständiger Regionalentwicklung

Influences and resources in the process of endogenous development

Influences et ressources du processus de développement endogène

Quelle: Mühlinghaus 2002: 125; Graphik: L. BaumanN 
zudem die Zusammenarbeit und die Bereitschaft der Bevölkerung zur Akzeptanz lokaler Initiativen.

In beiden Fallbeispielen hat sich gezeigt, dass eigenständiger Regionalentwicklung durch externe Einflüsse Grenzen gesetzt sind. Die Weltwirtschaftslage, die europäische Integration sowie nationale und kantonale Vorschriften prägen die lokale Situation, können aber mit lokalen Handlungsansätzen kaum beeinflusst werden. Sie führen deshalb oft zu Resignation und schränken den lokalen Handlungsspielraum ein.

\subsection{Auswirkungen und Chancen eigenständiger Regionalentwicklung}

Obwohl einige lokale Initiativen neue Arbeitsplätze schaffen oder neue Einkommensquellen erschliessen, müssen die wirtschaftlichen Auswirkungen eigenständiger Regionalentwicklung als bescheiden beurteilt werden. Die wirtschaftliche Situation peripherer Berggebiete wird zu stark durch externe Einflüsse geprägt. Die Hauptauswirkungen eigenständiger Regionalentwicklung liegen im soziokulturellen Bereich. Die Fallstudien haben gezeigt, dass das Bewusstsein der Bevölkerung für lokale Probleme sowie die regionale Identität und der soziale Zusammenhalt wachsen. Dies kann indirekt auch die wirtschaftliche Entwicklung positiv beeinflussen, indem dadurch innovative Akteure motiviert werden, in ländlichen Gebieten zu bleiben und sich für lokale Anliegen zu engagieren.

Eigenständige Regionalentwicklung ist eine Chance für periphere Gebiete, weil sie einen eigendynamischen und emanzipatorischen Entwicklungsprozess auslösen kann (Mose 1993: 199). In lokalen Initiativen können Akteure neue Kontakte knüpfen und Erfahrungen sammeln, was ihnen in anderen Bereichen später zugute kommt. Zudem können erfolgreiche Projekte eine positive Grundstimmung in der Bevölkerung verbreiten und zur Nachahmung animieren.

Um diese Chancen eigenständiger Regionalentwicklung besser wahrnehmen zu können, muss ein Umdenken stattfinden. Allzu oft werden die Erfolge von Entwicklungsprozessen anhand klar messbarer Endresultate beurteilt. Neben den Resultaten sollte aber auch dem Entwicklungsprozess vermehrt Aufmerksamkeit geschenkt werden (vgl. SHORTALl \& SHUCKSMITH 1998: 83). Eigenständige Regionalentwicklung kann einen dynamischen, kreativen und bedürfnisgerechten Prozess in Gang setzen, was für periphere Gebiete genauso wichtig sein kann wie neu geschaffene Arbeitsplätze oder eine Erhöhung touristischer Übernachtungszahlen.

\subsection{Ansätze zur Förderung eigenständiger Regionalentwicklung}

Die Resultate der Fallstudien und der Expertengespräche zeigen, dass in der Förderung eigenständiger
Regionalentwicklung verschiedene Akteure zusammenwirken müssen. Der Bund hat mit den Instrumenten Regio Plus, Innotour, Interreg und der landwirtschaftlichen Absatzförderung Möglichkeiten zur finanziellen Unterstützung von Basisinitiativen geschaffen. Wichtige Animations-, Promotions- und Moderatorenaufgaben übernehmen die regionalen Geschäftsstellen (RÜEGG \& KLÖTI 2000:24). Durch ihre Position zwischen privatem und öfentlichem Sektor sowie zwischen Gemeinden und Bund bzw. Kantonen können sie wichtige Vermittlungsfunktionen für Informationen, Beratungsangebote, finanzielle Mittel und Kooperationspartner übernehmen. Dadurch spielen sie eine wichtige Rolle in der eigenständigen Regionalentwicklung und sollten in der Wahrnehmung dieser Aufgabe vermehrt unterstützt werden.

Gemeinden sollten diejenigen Aufgaben übernehmen, welche eine grosse Bürgernähe verlangen wie die Stärkung des sozialen Zusammenhaltes oder die Förderung der Informationsverbreitung über lokale Belange. Weiter sollten sie Massnahmen zur besseren Integration von Neuzuzügern ergreifen, weil diese vielfach eine wichtige Rolle im Entwicklungsprozess übernehmen.

Wenn es um die Finanzierung lokaler Initiativen geht, sind auch private Akteure und Nichtregierungsorganisationen gefragt. Durch die Unterstützung partizipativer, den regionalen Bedürfnissen entsprechender, auf endogenen Potentialen aufbauender und den natürlichen Gegebenheiten angepasster Projekte können auch sie einen Beitrag leisten.

Ein Grundproblem der Förderung eigenständiger Regionalentwicklung besteht darin, dass Geldgeber meist innerhalb kurzer, klar vorgegebener Frist eindeutig messbare Resultate benötigen. Die Auswirkungen eigenständiger Regionalentwicklung hingegen bestehen oft aus längerfristigen Prozessen und sind vielfach nicht messbarer Natur. Dadurch werden sie oft zu wenig wahrgenommen.

\section{Schlussfolgerungen und Diskussion}

Die vorliegenden Resultate zeigen, dass eigenständige Regionalentwicklung in ländlich peripheren Gebieten einen eigendynamischen Entwicklungsprozess auslösen kann und so eine Chance für diese darstellt. Aufgrund der begrenzten wirtschaftlichen Auswirkungen sollte sie aber als Ergänzung und nicht als Ersatz traditioneller, exogener Strategien verstanden werden. Von besonderer Bedeutung für die eigenständige Regionalentwicklung ist, dass eine Kombination interner und externer Ressourcen gesucht wird. Die vorliegende Untersuchung gibt einige Hinweise, wie eine 
solche aussehen könnte (vgl. Mühlinghaus 2002). Beispielsweise sind oft externe finanzielle Mittel, Beratungen oder Informationen notwendig, um lokale Initiativen auszulösen oder erfolgreich umzusetzen. Es braucht folglich häufig externe Ressourcen, um die endogenen Potentiale zu aktivieren. Umgekehrt können lokale Initiativen dazu beitragen, das regionale Selbstbewusstsein zu stärken und die Kompetenzen der Akteure zu verbessern, wodurch periphere Gebiete ihre Interessen gegen aussen besser vertreten und vermehrt von externen Ressourcen profitieren können.

\section{Dank}

Bedanken möchte ich mich an dieser Stelle bei der Stiftung für wissenschaftliche Forschung an der Universität Zürich für die finanzielle Unterstützung der Feldarbeit.

\section{Literatur}

Barke, M. \& M. Newton (1994): A New Rural Development Initiative in Spain: The European Community’s «Plan Leader». - In: Geography 79/4: 366-371.

BASSAND, M. (1986): The Socio-Cultural Dimension of Self-Reliant Development. - In: BASSAND, M. et al. (Hrsg.): Self-Reliant Development in Europe. Theory, Problems, Actions. - Aldershot: Gower: 130-144.

Birner, A., Fried, H. \& A. Novy (1997): Lokale Entwicklungsinitiativen. Ein interkultureller Vergleich. Lebensstrategien und globaler struktureller Wandel. Frankfurt am Main: Lang.

BRUCKMEIER, K. (2000): LEADER in Germany and the Discourse of Autonomous Regional Development. In: Sociologia Ruralis 40/2: 219-227.

Brugger, E.A. (1984): Endogene Entwicklung: Ein Konzept zwischen Utopie und Realität. - In: Informationen zur Raumentwicklung 1984/1-2: 1-19.

Brugger, E.A. (1986): Endogenous Development: A Concept between Utopia and Reality. - In: BASSAND, M. et al. (Hrsg.): Self-Reliant Development in Europe. Theory, Problems, Actions. - Aldershot: Gower: 38-58. Bryden, J. (1998): Lokale Antworten auf globale Trends. - www.rural-europe.aeidl.be/rural-de/biblio/ global/art01.html, 21.12.1998.

BundeSAMT FÜR STATISTIK (1998a): Eidgenössische Betriebszählung 1995. Die Gemeinden im Vergleich 1985/1991/1995. Arbeitsstätten und Beschäftigte nach ausgewählten Wirtschaftsarten. - Bern: Bundesamt für Statistik.

BUNDESAMT Für STATISTIK (1998b): Eidgenössische Landwirtschaftszählung 1996. - Bern: Bundesamt für Statistik.

Dusmovirs, R. (1996): Eigenständige Entwicklung in ländlich-peripheren Regionen. - Frankfurt: Lang.

Elsasser, H. (1987): Regionalismus und endogene
Entwicklung in der Schweiz. - In: Agrarische Rundschau 6: 3-6.

Farrell, G., Thirion, S. \& P. Soto (1999): Territoriale Wettbewerbsfähigkeit. Der Entwurf einer gebietsbezogenen Entwicklungsstrategie unter Berücksichtigung der Erfahrungen aus LEADER. - In: Innovation im ländlichen Raum Heft Nr. 6, Band 1. - Brüssel: Europäische Beobachtungsstelle LEADER.

FLICK, U. (1996): Qualitative Forschung. Theorie, Methoden, Anwendung in Psychologie und Sozialwissenschaften. - Hamburg: Rowohlt Taschenbuch.

FüRST, D. (2000): Eigenständige Regionalentwicklung im Zeichen der Globalisierung. - In: Tourismus Journal 4/2: 177-194.

Glaser, B.G. \& A.R. Strauss (1998): Grounded Theory. Strategien qualitativer Forschung. - Bern: Verlag Hans Huber.

Glatz, H. \& G. Scheer (1981): Eigenständige Regionalentwicklung. Ein Weg für strukturell benachteiligte Gebiete in Österreich. - = Schriftenreihe des Bundeskanzleramtes, Sektion IV/Abt. 6, Wien: Bundeskanzleramt/Bundespressedienst.

HAHNe, U. (1985): Regionalentwicklung durch Aktivierung intraregionaler Potentiale. $-=$ Schriften des Instituts für Regionalforschung der Universität Kiel, Band 8, Kiel: Verlag V. Florentz.

Hahne, U. (1987): Endogene Regionalentwicklung. Ansatz zwischen ökonomischen Fallgruben, Historismus und Pragmatismus. - In: Bremer Beiträge zur Geographie und Raumplanung 11: 401-416.

HartKe, S. (1985): Koordinations- und Kooperationsprobleme einer Entwicklungspolitik für Regionen: Welche Chancen hat die eigenständige Regionalentwicklung in der Bundesrepublik Deutschland. - In: Stuckey, B. (Hrsg.): Endogenous Development. - = Nationales Forschungsprogramm «Regionalprobleme in der Schweiz», Arbeitsberichte, Bern: Programmleitung NFP «Regionalprobleme».

Heintel, M. (1998): Einmal Peripherie - immer Peripherie? Szenarien regionaler Entwicklung anhand ausgewählter Fallbeispiele. - = Abhandlungen zur Geographie und Regionalforschung, Band 5, Wien: Institut für Geographie.

HoffmanN, N. \& H. Nuissl (1998): Zwischen Halbgott und Handlanger. Zum Akteursverständnis in Konzepten der eigenständigen Regionalentwicklung. - In: Kujath, H.J. (Hrsg.): Strategien der regionalen Stabilisierung: wirtschaftliche und politische Antworten auf die Internationalisierung des Raumes. - Berlin: Edition Sigma: 251-289.

LAMNEK, S. (1995a): Qualitative Sozialforschung, Band 1: Methodologie. - 3., korrigierte Auflage, Weinheim: Beltz Psychologieverlagsunion.

LAMNEK, S. (1995b): Qualitative Sozialforschung, Band 2: Methoden und Techniken. - 3., korrigierte Auflage, Weinheim: Beltz Psychologieverlagsunion.

MAYRING, P. (2000): Qualitative Inhaltsanalyse. Grund- 
lagen und Techniken. - 7. Auflage, Weinheim: Deutscher Studienverlag.

MosE, I. (1989): Eigenständige Regionalentwicklung - Chance für den peripheren ländlichen Raum? - In: Geographische Zeitschrift 77/3: 154-167.

MosE, I. (1993): Eigenständige Regionalentwicklung - neue Chancen für die ländliche Peripherie? - = Vechtaer Studien zur Angewandten Geographie und Regionalwissenschaften, Band 8, Vechta: Vechtaer Druck und Verlag.

MüHLINGHAus, S. (2002): Eigenständige Regionalentwicklung im Schweizer Berggebiet - Umsetzungsprozesse, Erfolgsaussichten und Förderansätze. - = Publikation der Ostschweizerischen Geographischen Gesellschaft, Neue Folge, Heft 7, St. Gallen: Ostschweizerische Geographische Gesellschaft.

Pongratz, H. \& M. KReIl (1999): Möglichkeiten einer eigenständigen Regionalentwicklung. - In: Zeitschrift für Agrargeschichte und Agrarsoziologie 39/1: 91-111. RAY, C. (1998): Territory, Structures and Interpretation - Two Case Studies of the European Union's LEADER I Programme. - In: Journal of Rural Studies 14/1: 79-87.

RAY, C. (2000): Endogenous Socio-Economic Development in the European Union - Issues of Evaluation. - In: Journal of Rural Studies 16: 447-458.

RüEGG, E. \& U. KLÖTI (2000): Politisch-institutionelle Aspekte der eidgenössischen Regionalpolitik (PIADER). Eine Auswertung der Erfahrungen von 3 IHG-Regionen. - = SECO-Publikation, Standortförderung Nr. 1 (2.2000), Bern: SECO.

Sauberer, M., Schindegger, F. \& F. Tödtling (1989): Strategien für entwicklungsschwache Problemgebiete. - = Schriftenreihe Nr. 77, Wien:Österreichische Raumordnungskonferenz (ÖROK).

SHORTALL, S. \& M. SHUCKSMITH (1998): Integrated Rural Development: Issues Arising from the Scottish Experience. - In: European Planning Studies 6/1:73-88.

Shucкsmith, M. (2000): Endogenous Development, Social Capital and Social Inclusion: Perspectives from LEADER in the UK. - In: Sociologia Ruralis 40/2: 208-218.

SleE, B. (1994): Theoretical Aspects of the Study of Endogenous Development. - In: VAN DER Ploeg, J.D. \& A. Long (Hrsg.): Born from within. Practice and Perspective of Endogenous Rural Development. - Assen: Van Gorcum.

STöHr, W.B. (1981): Development from Below: The Bottom-Up and Periphery-Inward Development Paradigm. - In: STÖHR, W.B. \& F. TAYLOR (Hrsg.): Development from Above or Below? The Dialectics of Regional Planning in Developing Countries. - Ann Arber: UMI: 39-72.

STöHR, W.B. (Hrsg.) (1992): Global Challenge and Local Response. Initiatives for Economic Regeneration in Contemporary Europe. $-2^{\text {nd }}$ edition, London: Mansell.
WiLson, P. (1995): Embracing Locality in Local Economic Development. - In: Urban Studies 32/4-5: 645-658.

\section{Zusammenfassung: Eigenständige Regionalentwick- lung als Strategie für periphere ländliche Räume?} Eine Untersuchung aus dem Schweizer Berggebiet In diesem Artikel wird auf Grundlage zweier Fallstudien aufgezeigt, inwiefern eigenständige Regionalentwicklung eine sinnvolle Strategie für ländlich-periphere Räume darstellt. Für eine erfolgreiche Umsetzung eigenständiger Regionalentwicklung bedarf es einer Kombination interner und externer Ressourcen sowie eines regen Informationsaustausches. Die Hauptauswirkungen eigenständiger Regionalentwicklung sind nicht im wirtschaftlichen, sondern im soziokulturellen Bereich zu sehen. Letztere können sich aber positiv auf die wirtschaftliche Entwicklung auswirken, indem sie innovative Akteure motivieren in peripheren Gegenden zu bleiben und sich für lokale Anliegen zu engagieren. Mit einer gezielten Förderung könnte der Beitrag eigenständiger Regionalentwicklung gestärkt werden. Von Bedeutung sind insbesondere die Bereitstellung von Beratungsangeboten und finanziellen Mitteln, die Vernetzung von Akteuren sowie die Stärkung des sozialen Zusammenhalts.

\section{Summary: Endogenous Development as Strategy for Peripheral Rural Regions? Survey of two Swiss mountain regions}

On the basis of two case studies, this paper explores endogenous development as a strategy for peripheral rural regions. The results have shown that successful endogenous development requires a combination of internal and external resources, as well as a continuous exchange of information. The main impacts of endogenous development are not in the economic, but in the socio-cultural realm. Changes in the latter can, however, indirectly have a positive impact on the regional economy, because innovative actors may be motivated to stay in the peripheral regions and to become involved in local development activities. Welldirected support could increase the impact of endogenous development. Consequently, peripheral regions should have access to financial resources and outside assistance. Additionally, networking among actors as well as the strengthening of social cohesion are important.

Résumé: Le développement endogène comme stratégie pour les régions rurales périphériques? Une étude des régions de montagne suisses

Le but de cet article est de démontrer les chances du développement endogène comme stratégie pour des régions rurales périphériques. Les résultats de deux études de cas ont fait apparaître clairement que 
dans tout processus de mise en œuvre une combinaison de ressources régionales et externes est indispensable ainsi qu'un échange continu d'informations. Les retombées principales du développement endogène ne se manifestent pas au niveau économique mais au niveau socioculturel. Pourtant, ces dernières peuvent influencer positivement le développement économique en incitant les acteurs porteurs de changement à rester en zone rurale et à s'engager dans des initiatives locales. La politique régionale devrait davantage tenir compte des mérites du développement endogène et soutenir financièrement les initiatives locales, créer des services de consultation adaptés au besoin régional et renforcer la cohésion sociale.

Dr. Sabine Mühlinghaus, Geographisches Institut der Universität Zürich, Winterthurerstr. 190, CH-8057 Zürich.

e-mail:muehling@bluewin.ch

Manuskripteingang/received/manuscrit entré le 19.11.2001

Annahme zum Druck/accepted for publication/accepté pour l'impression: 23.5.2002 